\title{
Retransmission Mechanism for Partially Reliable Transport Protocols Based on Multimedia Applications Transmission
}

\author{
Ching-Neng Lai and Yen-Hung Chen
}

\begin{abstract}
The partially reliable transport protocol can enhance the quality of services $(\mathrm{QoS})$ of multimedia applications because it reliably transmits some packets and unreliably transmits other packets. Nevertheless, determining which packets require reliable, i.e., these packets will be retransmitted if they were lost, significantly influences the performance of multimedia applications. For the determination, existing approaches adopt a fixed threshold or require information from other layers. This paper proposes a Timeliness-aware Retransmission Mechanism (TRM) that dynamically determines which lost packets should be retransmitted without any information provided from other layers. The receiver using TRM determines a threshold, which is the packet sequence number having timeliness, according to the packet consumed rate and the Round-Trip Time (RTT). Thus the sender retransmits lost packets that can timely arrive, namely, the packets have sequence numbers larger than this threshold. Simulation results shows that PR-DCCP, a partially reliable transport protocol, with TRM can efficiently increase the Decodable Frame Ratios (DFRs), which represents QoS, and decrease the Useless Data Received Ratios (UDRRs), which represents the bandwidth waste. In the simulations, TRM can achieve 8.6 10.6\% DFR improvement and 15.1\% 17.6\% UDRR improvement on average, compared with other retransmission methods.
\end{abstract}

Index Terms-Timeliness-aware retransmission, multimedia applications, partial reliability transmission.

\section{INTRODUCTION}

The rapid development of multimedia applications has changed the daily life of many people. Users can enjoy the multimedia applications, such as Voice over Internet Protocol (VoIP), Video-on-Demand (VoD) and Internet Protocol TV (IPTV), everywhere via Internet. However, a volatile network environment inevitably causes packet losses. The losses of certain key packets cause that the receiver only receives the fragmented data, and even invalidate numerous successfully received packets that depend on the lost packets. This results in a massive amount of packets being rendered unusable, despite being successfully received by the receiver. For instance, if the I-frame packets in an MPEG file are lost, the corresponding $\mathrm{P}$ - and $\mathrm{B}$-frame packets are unworkable even if they are successfully received. Thus, if the key packets for an

Manuscript received April 24, 2015; revised October 12, 2015.

Ching-Neng Lai is with the Department of Information Technology, Hsing Wu University, New Taipei City, 24452, Taiwan (e-mail: 093062@mail.hwu.edu.tw).

Yen-Hung Chen is with Cyber Trust Technology Institute, Institute for Information Industry, Taipei, 10622, Taiwan (e-mail: pplong@gmail.com). application can be retransmitted after being lost, the QoS of multimedia applications would be significantly improved. Based on the above, the partially reliable transport protocol, which can reliably transmit some packets and unreliably transmit other packets, is suitable to multimedia applications. Currently there are two main partially reliable transport protocols: PR-DCCP [1], the partial reliability extension of Datagram Congestion Control Protocol (DCCP) [2]; and PR-SCTP [3], the partial reliability extension of Stream Control Transmission Protocol (SCTP) [4].

In using partially reliable transport protocols for multimedia applications, the main issue is how the sender can accurately determine which lost packets should be retransmitted. If the sender aborts retransmission of lost packets that can be valid, resulting data is incomplete. On the other hand, repeatedly retransmitting out-of-date packets generates inefficient bandwidth utilization.

The methods to determine which lost packets should be retransmitted can be classified into three categories: (1) the sender fixes the number of retransmission times for lost packets. That is, all lost packets are retransmitted, and the retransmission of a lost packet is aborted if its number of times exceeds a given threshold [5]; (2) the sender fixes packet lifetime based on the characteristics of multimedia applications. This value is generally based on experience or on experimental data [6], [7]; (3) the sender dynamically determines packet lifetime based on information of the other upper layer protocols [8]-[10].

However, the decisions of retransmitting lost packets in categories (1) and (2) are made according to predetermined values of retransmission times and packet lifetime, respectively. In a volatile network, the network conditions under retransmissions may substantially differ from the network conditions under which the predetermined value was originally set. As such, the predetermined value may not fulfill the requirements on retransmissions. On the other hand, the methods in category (3) need to adopt the particular upper layer protocols.

The retransmission method should consider the timeliness of retransmitted packets so that it not only ensures useful retransmissions to the receiver, but also prevents invalid retransmissions. Thus this paper proposes a Timeliness-aware Retransmission Mechanism (TRM) that dynamically determines which lost packets should be retransmitted without information from other layers. The receiver using TRM determines a threshold, which is the packet sequence number having timeliness, according to the packet consumed rate and the RTT. According to this threshold, the sender 
retransmits packets that can timely arrive, namely, the packets have sequence numbers larger than this threshold.

The contributions in this paper have three points. First, this paper is the first one to investigate different retransmission methods by using PR-DCCP. Second. The proposed TRM uses the statistical method to confirm that a certain percentage of retransmissions is timely. Third, TRM can enhance better performance of multimedia applications than other retransmission methods.

This paper is organized as follows. Section II first describes two partially reliable transport protocols, including PR-SCTP and PR-DCCP. Related works of retransmission methods are also described. The proposed TRM approach is formally described in Section III. Section IV discusses simulation results to compare the performance of TRM with that of other retransmission methods. Finally, conclusions and future works are given in Section V.

\section{BACKGROUND}

\section{A. $P R-D C C P$}

PR-DCCP, an extension of unreliable DCCP, encounters more difficulties for several reasons [1]. First, DCCP uses an incremental sequence number. Thus, the original sequence number cannot be used for packet retransmission. Second, the sender must inform the receiver which packets require reliable delivery. To solve the first problem, PR-DCCP adopts sequence number compensation, which adds an offset into the retransmitted packet, so that the receiver can calculate the original sequence number by using both the new sequence number and the offset. Further, PR-DCCP solves the second problem by using a new DCCP option to record information if the packet requires reliable delivery. Essentially, the sender using PR-DCCP retransmits the packet with the new sequence number and an offset. After receiving a retransmitted packet, the receiver uses the new sequence number and the offset to recognize its original sequence number.

Given a same environment, PR-SCTP and PR-DCCP achieve similar QoS in multimedia applications [1]. However, PR-DCCP has less overhead than PR-SCTP, so the former is more suitable to multimedia applications. This study therefore applied PR-DCCP for evaluating the proposed TRM.

\section{B. Related Works}

The methods to determine which lost packets should be retransmitted can be classified into three categories. The first category is that the sender fixes the number of retransmission times for the packets. The scheme proposed in [5] transmits lost packets within a fixed number of times in a specific multimedia stream with PR-SCTP. The second category is that the sender fixes packet lifetime based on the characteristics of multimedia applications. In [6], MPEG-4 films are transmitted by PR-SCTP in a FreeBSD system. The packet lifetime is fixed according to experimental results. Additionally, this scheme only retransmits lost packets of I-frame, but does not retransmit lost packets of $\mathrm{P}$ - and B-frames. In [7], the sender further retransmits lost packets of $\mathrm{I}-, \mathrm{P}-$ and B-frames, but each type is assigned an individual lifetime according to experimental results.
The third category is that the sender dynamically determines packet lifetime based on the characteristics of upper layer protocols. In [8], PR-SCTP is used as the transport protocol for transmitting SIP messages to avoid redundant transmission, packet lifetime in PR-SCTP is determined by the timeout value of SIP. Some researches allow the receiver to use RTP and RTCP to transmit playout time of packets to the sender, so the sender knows when each packet is being played out at the receiver [9], [10]. By estimating RTT and receiving playout time of each packet, the sender can determine whether the retransmitted packets can timely arrive at the receiver.

However, in a volatile network, the network conditions under retransmissions may substantially differ from the network conditions under which the predetermined value was originally set. As such, the predetermined value may not fulfill the requirements on retransmissions because of two cases. If this value is too small, the sender abandons retransmitting the lost packets although these packets are still valid. If this value is too large, the sender continues retransmitting useless packets. On the other hand, the approaches utilizing information of upper layer protocols require specific protocols. Thus, they are not suitable for all kinds of multimedia applications. Also the cross layer handling required for these approaches increases the complexity of their implementation.

\section{TIMELINESS-AWARE RETRANSMISSION MECHANISM}

\section{A. Operation}

Fig. 1 illustrates the basic operation of TRM. When the receiver receives the $i$-th packet, $P_{i}$, it transmits the acknowledgement (Ack) packet which contains $T H_{i}$ to the sender. Determining proper $T H_{i}$ is based the timeliness of retransmission. The receiver will estimate the next RTT and how many packets will be consumed during this RTT. Thus the receiver can guess which packet will be consumed right after the RTT. However, directly using the sequence number of this packet is not satisfactory because many retransmissions are not timely due to the estimation errors. Thus, the statistical method is adopted to calculate proper $T H_{i}$ to let most retransmitted packets be timely.

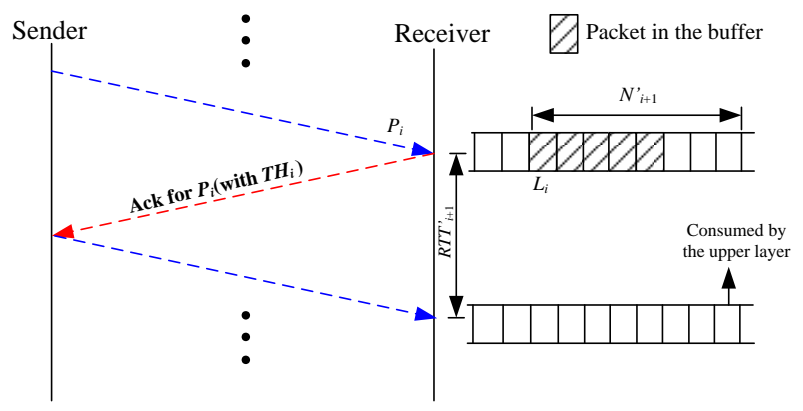

Fig. 1. The operation of TRM.

\section{B. Estimation of RTT and the Number of Consumed Packets}

To correctly determine $T H_{i}$, the RTT and the packet 
consumed rate in the receiver's buffer should be estimated. First, the receiver must measure the current RTT. In DCCP or PR-DCCP, when the sender receives the Ack packet from the receiver, the sender attaches the sequence number of the Ack packet to the Ack of Ack packet and then, by transmitting the packet, informs the receiver that the Ack packet has been received. Thus, the receiver can use the sequence number of the Ack packet to obtain the current RTT. Let $R T T_{i}$ denote the RTT measured by the receiver at receiving the packet $P_{i}$. Then the estimated next RTT, $R T T_{i+1}^{\prime}$, at this instant can be obtained by using an exponential smoothing method as

$$
R T T_{i+1}^{\prime}=\frac{1}{8} R T T_{i}+\frac{7}{8} R T T_{i}^{\prime} .
$$

About the packet consumed rate, the receiver also measures how many packets are consumed during $R T T_{i}$ at receiving the packet $P_{i}$. Let $N_{i}$ denote the number of consumed packets in the receiver's buffer during $R T T_{i}$. Thus the packet consumed rate, $R_{i}$, during $R T T_{i}$ is computed as

$$
R_{i}=N_{i} / R T T_{i}
$$

To minimize the error in estimating $R_{i+1}^{\prime}$, we use the trend-adjusted exponential smoothing method, rather than the exponential smoothing method. The trend-adjusted exponential smoothing method considers two factors: the smoothing error between the previous and current calculations, as well as the trends in previous calculations, thus significantly eliminating the irregular elements within the trend effect. Adopting this method, the receiver calculates packet consumed rate by

$$
R_{i+1}^{\prime}=S_{i}^{\prime}+T_{i}^{\prime}
$$

where $S_{i}^{\prime}$ and $T_{i}^{\prime}$ represent the estimation of the packet consumed rate and its trend, respectively. With the exponential smoothing method, the calculations of $S_{i}^{\prime}$ and $T_{i}^{\prime}$ are shown as

$$
\begin{gathered}
S_{i}^{\prime}=0.3 R_{i}+0.7 R_{i}^{\prime}, \\
T_{i}^{\prime}=\alpha\left(R_{i}^{\prime}-R_{i-1}^{\prime}\right)+(1-\alpha) T_{i-1}^{\prime} .
\end{gathered}
$$

The value 0.3 is generally used as the smoothing parameter in Eq. (4), and the smoothing parameter $\alpha$ in Eq. (5) is usually set to less than or equal to 0.3 . A better $\alpha$ will be obtained by performing simulations.

Using Eqs. (1) and (3), the number of consumed packets, $N_{i+1}^{\prime}$, during $R T T_{i+1}^{\prime}$ can be estimated as

$$
N_{i+1}^{\prime}=R T T_{i+1}^{\prime} \cdot R_{i+1}^{\prime}
$$

Let $L_{i}$ be the sequence number of the first packet stored in the receiver buffer. Thus the packet with sequence number $L_{i}+N_{i+1}^{\prime}$ will be consumed right after the RTT. However, setting this value to $T H_{i}$ will generate two problems. First,
$N_{i+1}^{\prime}$ is an average value, so half retransmissions will be timely, but half retransmissions will not. Second, the estimation errors also raise the probability that retransmissions are invalid.

\section{Threshold Setting}

First, we use the mean absolute derivation (MAD) to obtain the standard derivation of $N$. Let $M A D_{i+1}^{\prime}$ denote the estimated next MAD of $N$ at receiving packet $P_{i}$, and it can be easily calculated by using the exponential smoothing method as

$$
M A D_{i+1}^{\prime}=0.3\left|N_{i}-N_{i}^{\prime}\right|+0.7 M A D_{i}^{\prime}
$$

For an estimation error following a normal distribution, a standard deviation of $\sigma$ is equal to $\sqrt{\frac{\pi}{2}} \times M A D_{i} \approx 1.25 M A D_{i}$ [11]. Thus, using cumulative distribution function (CDF) of a normal distribution with mean $=\mu$ and variance $=\sigma^{2}$, the probability exceeding $95 \%$ can be calculated as

$$
\begin{aligned}
& \Phi_{u, \sigma^{2}}(Z)=\frac{1}{\sqrt{2 \pi \sigma^{2}}} \int_{-\infty}^{Z} \exp \left(-\frac{(x-u)^{2}}{2 \sigma^{2}}\right) d x=0.95 \\
& \Rightarrow Z=u+1.65 \sigma .
\end{aligned}
$$

Therefore, when we expect that $95 \%$ retransmissions are timely, we should set the threshold $T H_{i}$ as

$$
\begin{aligned}
T H_{i} & =L_{i}+N_{i+1}^{\prime}+1.65 \sigma \\
& =L_{i}+N_{i+1}^{\prime}+2.0625 M A D_{i+1}^{\prime}
\end{aligned}
$$

\section{Simulation AND Evaluation}

\section{A. Simulation Environment}

Using NS2 [12], we simulated different retransmission methods to evaluate their performance in transmitting multimedia data. Fig. 2 shows the network topology used in the simulation. The router adopts drop-tail queue management. Connection 1 (Sender 1 - Receiver 1) uses PR-DCCP (TRM), PR-DCCP (fixed lifetime), DCCP and TCP, separately, while the other connection (Sender 2 Receiver 2) adopts TCP. DCCP is used as a baseline because it only executes unreliable transmission. Beside, in order to easily see the contributions of this work, we use TCP as the representative of the reliable transport protocols. Since our previous work [1] shows that the characteristics of data transmission of reliable transport protocols are very similar, the simulation results of other reliable protocols, such as SCTP, are close to that of TCP. Thus comparing our approach with TCP, a representative of reliable transport protocols, seems enough. The simulations focused on methods that use the information only obtained in the transport layer. Thus we do not compare the approaches which require extra information obtained from upper-layer protocols, such as SIP.

To evaluate the performance of multimedia applications, many kinds of data format can be used as the inputted sources 
for NS2. One of the sources is the raw video data transmitted by the EvalVid [13] - a framework and tool-set for evaluating the quality of video transmitted over a real or simulated communication network, such as the NS2. The researchers use the simulation results to calculate the Peak Signal-to-Noise Ratio (PSNR) and evaluate the quality of video images.

The MPEG-4 traffic trace file is another source which is a text document and composed of I, P, and B frames. Furthermore, the sizes and playout time of the frames are also recorded in the file. The researchers use the simulation results to measure the QoS parameters of the network, like loss rates, delay, and jitter. Since our research focuses on whether the retransmitted packets arriving in time or not and the completeness of the received data, the MPEG-4 traffic trace files were adopted as the sources [14]. The data sources were injected into the sending agent of NS2, the traffic trace files are directly adopted without any extra framework.

The bandwidth setting in the simulation depends on the bandwidth requirements of the trace files. Since the required bandwidth of the selected trace files used in our simulations were not large, the link capacity of the simulation topology in Fig. 2 was set from $0.1 \mathrm{Mbps}$ to $1 \mathrm{Mbps}$.

A group of I, P, and B frames passing through a standard encoding process becomes a GOP (Group of Pictures) [15]. During period of the decoding process, some data are useful, while some are useless. According to the data usability, two metrics, "Decodable Frame Ratio" (DFR) and "Useless Data Received Ratio" (UDRR) [16], were adopted to examine the efficiency of these retransmission methods.

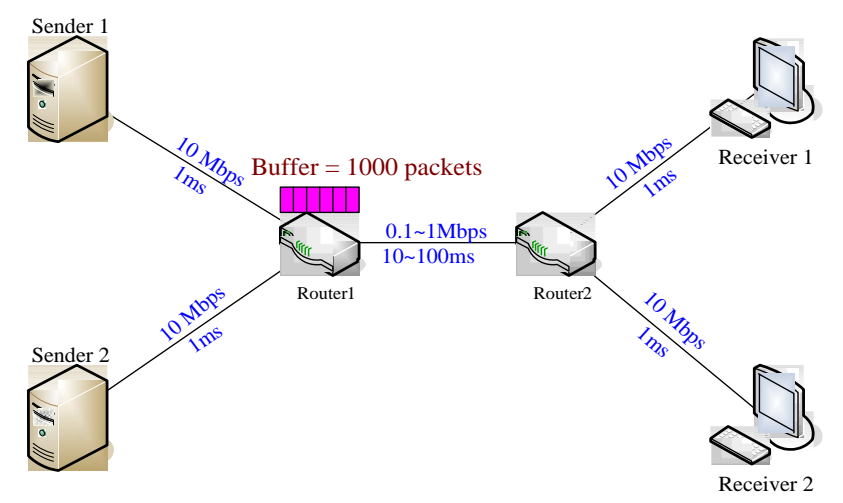

Fig. 2. Simulation topology.

DFR is defined as a percentage of the received effective frames (EF), which can be decoded into an effective GOP, among the total frames in the traffic trace file. When the packets received by the receiver can be decoded into a complete frame, this frame is called a "directly decodable frame". However, a directly decodable frame does not represent an effective frame. Apart from being directly decodable by itself, an EF needs to refer to frames that are also directly decodable during the decoding process. A higher DFR indicates a more satisfactory QoS.

UDRR is the percentage of useless packets among all received packets. One of the reasons of causing useless packets is the loss of packets in a frame or packets that may have been received but exceeded their lifetime, so that the received packets cannot be decoded into a directly decodable frame. As a result, they become useless data. Another reason is that the received frame is unable to refer to dependent frames while being decoded, thus causing this received frame to be useless. A higher UDRR means that more network resources are wasted.

The difference between DFR and UDRR is that the former computes the percentage of the useful frames occupying entire frames, but UDRR calculates the percentage covered by useless packets among all received packets. Given a scenario of two connections of identical throughput (i.e., the same number of received packets), a larger UDRR implies a smaller DFR. However, given a scenario of two connections of different throughput, this relationship is not necessarily true. For instance, since a connection utilizes larger bandwidth, it may simultaneously possess a larger DFR and a larger UDRR.

In the simulation, the different bandwidth and propagation delay were simulated to test the adaptability of TRM in a volatile network. Furthermore, different kinds of MPEG-4 traffic trace files were imported to understand the capability of TRM. Finally, the coexistence of different retransmission methods was also examined to perceive the applicability of TRM.

\section{B. The Effect of Bandwidth}

The simulation shows how different retransmission methods affect multimedia applications under different bandwidth. Packet lifetime was set to fixed values of 2 seconds and 0.2 seconds to assess the effects of large and small packet lifetime, respectively, on multimedia applications. Fig. 3 shows the simulation results. When bandwidth increases, the DFRs of all retransmission methods increase and their UDRRs decrease. PR-DCCP (TRM) obviously outperforms other methods. PR-DCCP (TRM) achieves a $10.6 \%$ DFR improvement over PR-DCCP $(\mathrm{LT}=0.2 \mathrm{~s})$ and an $8.6 \%$ improvement over PR-DCCP(LT $=2 \mathrm{~s})$ on average. On the other hand, PR-DCCP (TRM) achieves a $17.6 \%$ UDRR improvement over PR-DCCP $(\mathrm{LT}=0.2 \mathrm{~s})$ and a $15.1 \%$ over PR-DCCP (LT=2s) on average.

Since the packet lifetime is small, PR-DCCP $(\mathrm{LT}=0.2 \mathrm{~s})$ resembles an unreliable transport protocol (DCCP). Thus DFR and UDRR of PR-DCCP (LT=0.2s) are similar to those of DCCP. However, some lost packets which can arrive in time are still retransmitted in PR-DCCP $(\mathrm{LT}=0.2 \mathrm{~s})$. Therefore, PR-DCCP (0.2s) has slightly better DFR and UDRR than DCCP.

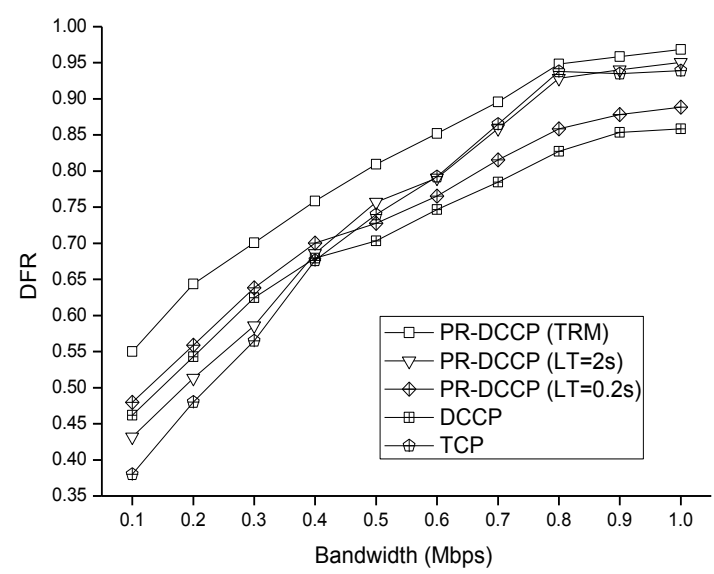

(a) 


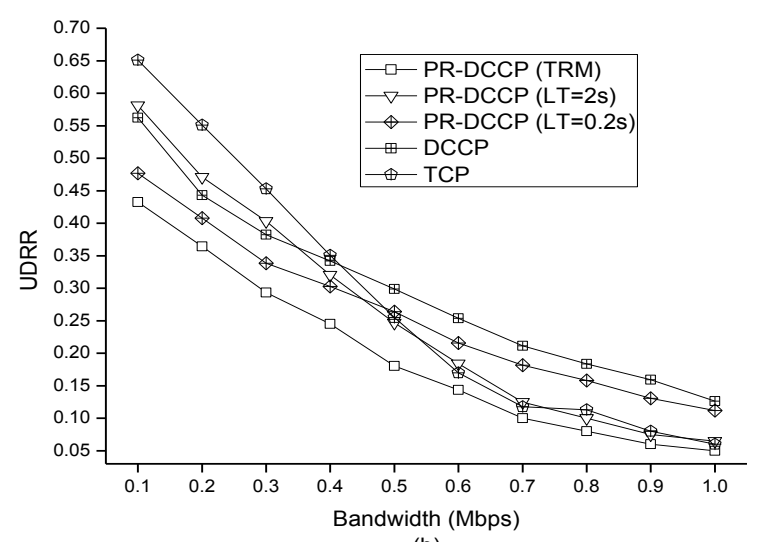

(b)

Fig. 3. DFR and UDRR versus link bandwidth.

Since the packet lifetime is large, the behavior of PR-DCCP (LT=2s) resembles that of TCP. The sender continues retransmitting lost packets until the lifetime of these packets is expired or the packets are received in the receiver. However, such packets may miss their effectiveness due to their losses of timeliness. Therefore, PR-DCCP $(\mathrm{LT}=2 \mathrm{~s})$ and TCP waste bandwidth at retransmitting useless packets. Under low bandwidth conditions, their DFR and UDRR values are worse than those obtained by other methods. Obviously, TCP has the worst DFR and UDRR because it is a reliable transport protocol. When bandwidth is larger, network congestion becomes lighter and more lost packets can timely arrive at the receiver, resulting in a higher DFR and a lower UDRR.

\section{The Effect of Propagation Delay}

The simulation shows how different retransmission methods affect multimedia applications under different propagation delay. Fig. 4 shows the simulation results. When the propagation delay increases, the DFRs of all retransmission methods decrease and their UDRRs increase. It is obvious that PR-DCCP (TRM) obviously outperforms other methods. PR-DCCP (TRM) achieves a 11.6\% DFR improvement over PR-DCCP (LT=0.2s) and a 7.8\% improvement over PR-DCCP ( $(\mathrm{T}=2 \mathrm{~s})$ on average. On the other hand, PR-DCCP (TRM) achieves an $8.7 \%$ UDRR improvement over PR-DCCP (LT=0.2s) and a $9.6 \%$ over PR-DCCP $(\mathrm{LT}=2 \mathrm{~s})$ on average.

Under conditions of short propagation delay, PR-DCCP $(0.2 \mathrm{~s})$ is significantly inferior to PR-DCCP (2s) and TCP. Small propagation delay actually increases the opportunity of retransmissions and the amount of effective retransmitted packets. However, setting an overly short packet lifetime still causes that the sender quickly stops retransmitting the lost packets, resulting in a poor DFR.

The figure also shows that PR-DCCP $(\mathrm{LT}=2 \mathrm{~s})$ and TCP generate good DFRs and UDRRs when propagation delay is small. This is because they always retransmit lost packets in this case and these packets are usually useful. As propagation delay increases, the probability that retransmitted packets become useless increases. However, PR-DCCP $(\mathrm{LT}=2 \mathrm{~s})$ and TCP still continue retransmitting invalid packets to the receiver, resulting in the increase of UDRRs. Also transmitting these useless packets consumes much bandwidth, result in the significant decrease of their DFRs. This phenomenon is especially critical in TCP, because PR-DCCP $(\mathrm{LT}=2 \mathrm{~s})$ stops retransmitting invalid packets when the lifetime of these packets is expired, but TCP does not.
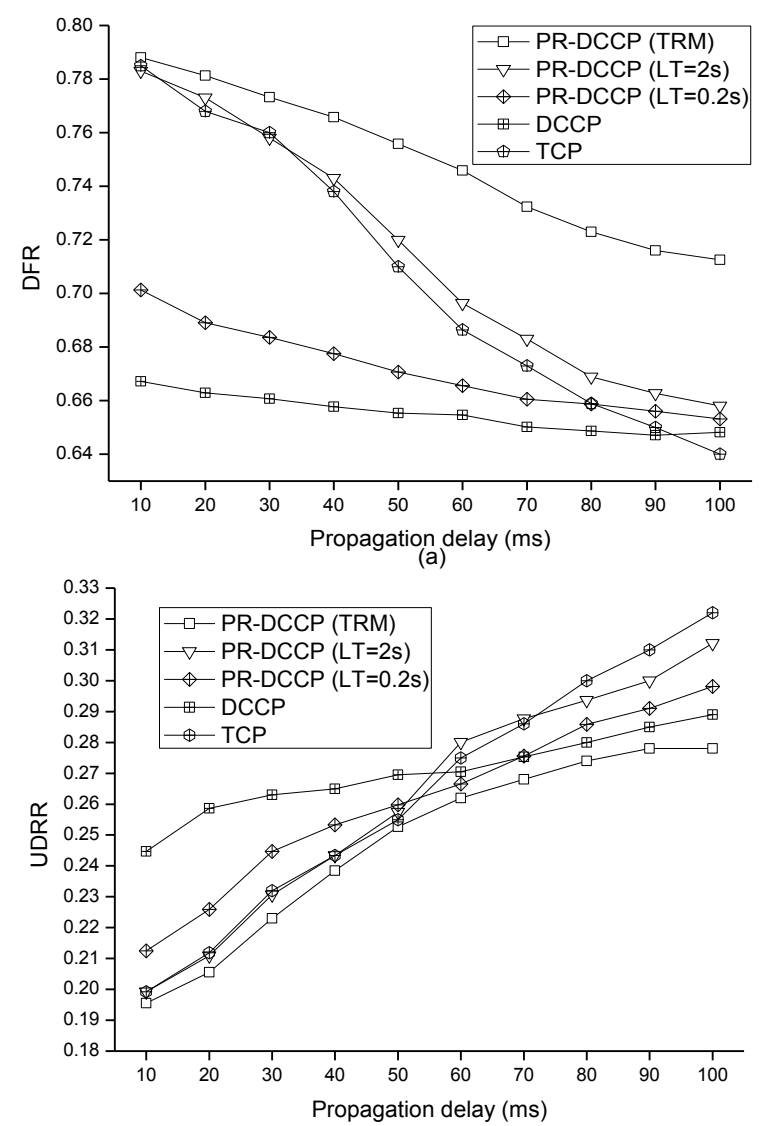

(b)

Fig. 4. DFR and UDRR versus link propagation delay.

\section{Different Videos}

In order to understand the capability of TRM for various types of films, we use six movies, "Silence of the Lambs", "Die Hard III", "Alpine Ski", "Star Trek-First Contact", "Robin Hood", and "Starship Troopers", as the source files. Their sizes are 254.69, 411.42, 363.476, 123.87, 313.8, and 271.17Mbytes. The movie "Die Hard III" has the largest volume of data and results in serious congestion. Therefore, it has the worst DFR and UDRR, as shown in Fig. 5. By contrast, the movie "Star Trek-First Contact", which has a relatively low volume of data, produces the best DFR and UDRR.

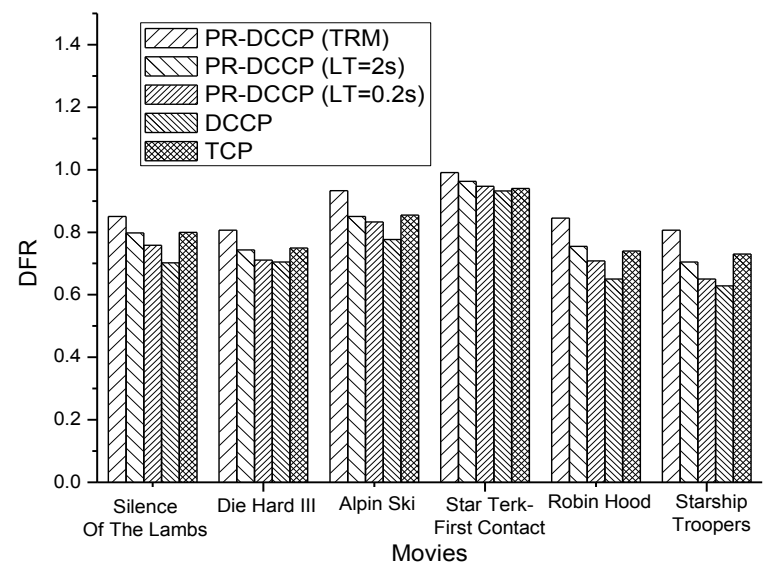

(a) 


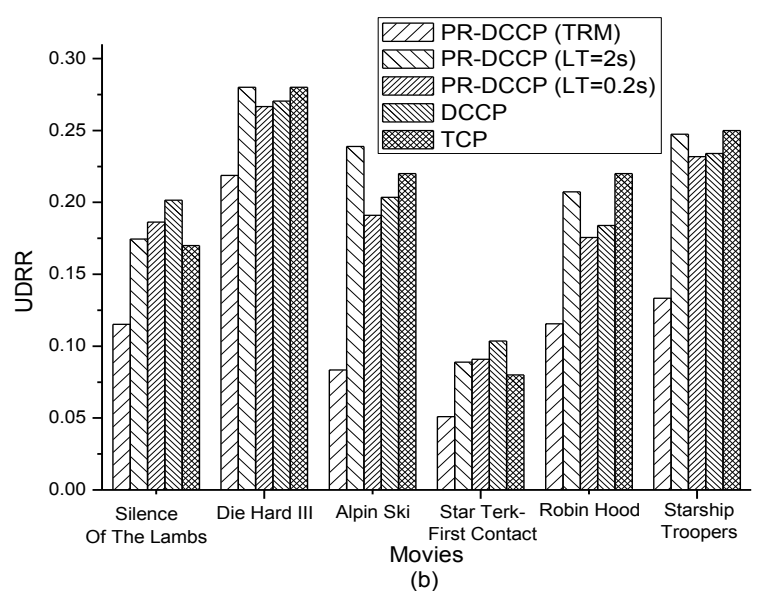

Fig. 5. DFR and UDRR for different films.

Regardless of which film we used, PR-DCCP (TRM) has a better DFR and UDRR than PR-DCCP (LT=2s), PR-DCCP (LT=0.2s), and TCP. Also DCCP has the worst DFR and UDRR. Although PR-DCCP (LT=2s) and TCP have higher DFRs than PR-DCCP (LT=0.2s) because the former retransmits more lost packets. However, some retransmitted packets may be useless, so PR-DCCP (LT=2s) and TCP produce worst UDRRs in some cases, i.e., the source is "Die Hard III", "Alpine Ski", "Robin Hood", or "Starship Troopers".

\section{E. Coexistence of Retransmission Methods}

The simulations above adopt a connection utilizing PR-DCCP (or DCCP), which competes with the other connection utilizing TCP. In this simulation, the results about the coexistence of different retransmission methods are observed. Fig. 6 shows the used network topology. The router uses the drop-tail queue management. Sender 1 to Receiver 1 uses PR-DCCP (TRM), Sender 2 to Receiver 2 uses PR-DCCP (LT=2s), Sender 3 to Receiver 3 uses PR-DCCP (LT=0.2s), Sender 4 to Receiver 4 uses DCCP, and Sender 5 to Receiver 5 uses TCP.

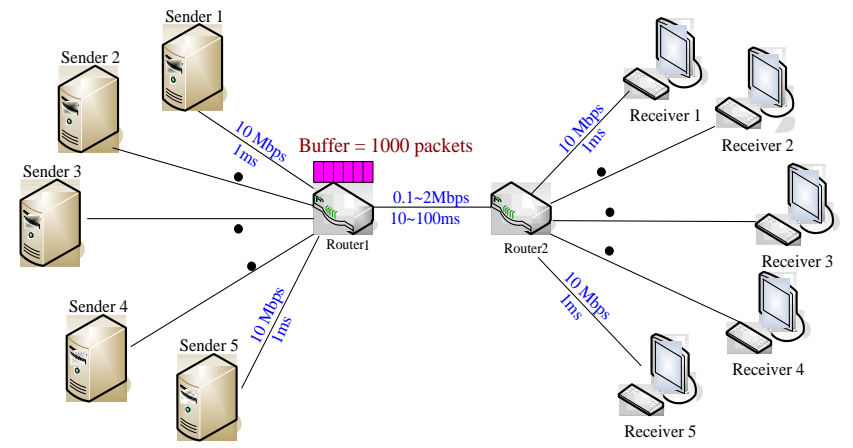

Fig. 6. Simulation topology for a coexisted environment.

Fig. 7 shows the results when different retransmission methods coexist under different bandwidth. As mentioned earlier, the characteristic of PR-DCCP $(\mathrm{LT}=2 \mathrm{~s})$ resembles that of TCP, while the characteristic of PR-DCCP $(\mathrm{LT}=0.2 \mathrm{~s})$ is similar to that of DCCP. Thus, PR-DCCP ( $\mathrm{LT}=2 \mathrm{~s})$ will retransmit many useless packets, while PR-DCCP $(\mathrm{LT}=0.2 \mathrm{~s})$ will not retransmit enough useful packets, resulting in their poor DFRs and UDRRs. Observed from this figure, PR-DCCP (TRM) obviously outperforms other retransmission methods when they coexist. Thus, DCCP
(TRM) can actually raise the QoS of multimedia applications in this more realistic environment.

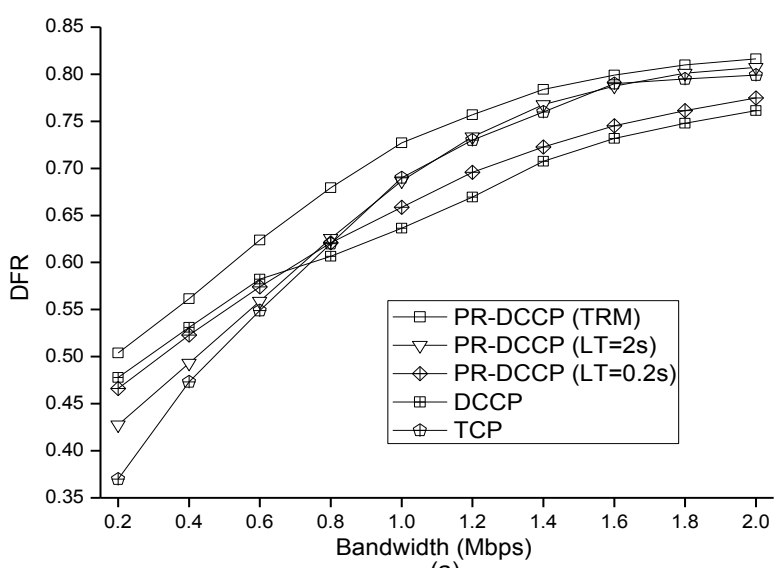

(a)

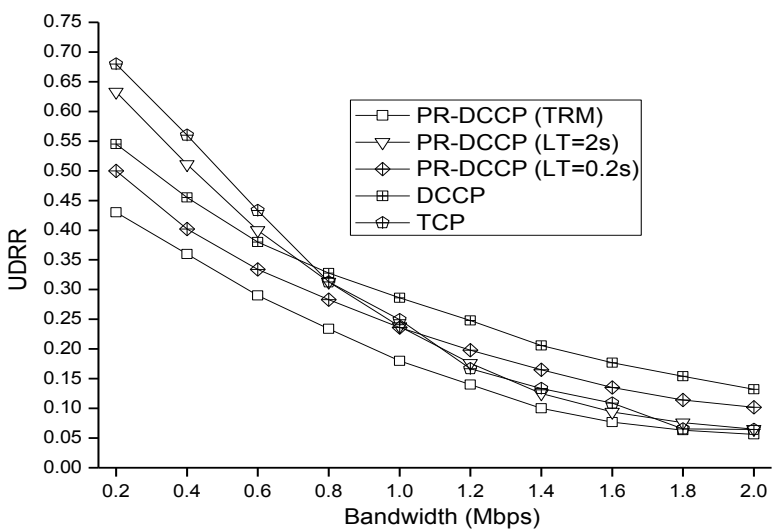

(b)

Fig. 7. DFR and UDRR versus link bandwidth under a coexisted environment.

\section{CONCLUSIONS AND FUTURE WORKS}

The partially reliable transport protocol will be widely used as the transport layer protocol for multimedia applications to improve their QoS. However, correctly deciding which packets should be retransmitted enhances not only the overall QoS of multimedia applications, but also the efficiency of bandwidth utilization. In our proposed TRM method, the receiver predicts the packet consumed rate and the RTT to estimate a threshold, which can confirm that 95\% retransmissions are timely. Then the sender will retransmit lost packets whose sequence numbers are larger than this threshold.

The simulation results show that TRM is superior to existing retransmission methods using fixed packet lifetime and TCP, regardless of how much bandwidth, what length of propagation delay, and what video file. Also in a coexisted environment, TRM still obtains a better DFR and UDRR than the others. This confirms that TRM actually provides better performance than other existing retransmission methods. Furthermore, implementing TRM only requires slight modifications for PR-DCCP.

In this study, the superiority of TRM is proven by using simulations. In the future, we will implement TRM and further observe its performance in a realistic network, including Delay Tolerant Network (DTN) [17], Long Time Evolution (LTE) network [18], and VANET [19]. 


\section{REFERENCES}

[1] Y. C. Lai and C. N. Lai, "DCCP partial reliability extension with sequence number compensation," Computer Networks, vol. 52, no. 16, pp. 3085-3100, 2008.

[2] S. Floyd, M. Handley, and E. Kohler, "Datagram congestion control protocol (DCCP)," IETF RFC 4340, March 2006.

[3] R. Stewart, M. Ramalho, Q. Xie, M. Tuexen, and P. Conrad, "Stream control transmission protocol (SCTP) partial reliability extension (PR-SCTP)," IETF RFC 3758, May 2004.

[4] R. Stewart, Q. Xie, K. Morneault, C. Sharp, H. Schwarzbauer, and T. Taylor, "Stream control transmission protocol (SCTP)," IETF RFC 2960, October 2000.

[5] L. Wang, K. I. Kawanishi, and Y. Onozato, "Simulation-based optimization on MPEG-4 over SCTP multi-streaming with differentiated retransmission policy in lossy link," in Proc. APSCC 2007, Tsukuba Science City, Japan, December 2007, pp. 164-171.

[6] M. Molteni and M. Villari, "Using SCTP with partial reliability for MPEG-4 multimedia streaming," in Proc. European BSD Conf., 2002, pp. 400-417.

[7] H. Wang, Y. Jin, W. Wang, J. Ma, and D. Zhang, "The performance comparison of PRSCTP, TCP and UDP for MPEG-4 multimedia traffic in mobile network," in Proc. ICCT 2003, Beijing, China, April 2003.

[8] X. L. Wang and V. Leung, "Applying PR-SCTP to transport SIP traffic," in Proc. IEEE Globecom., November 2005.

[9] H. Hagino, Y. Miyazaki, Y. Onoe, Y. Atsumi, H. Komaki, M. Taniguchi, and N. Yamanouchi, "A playout time oriented retransmission scheme for multimedia streaming systems," High-Speed Networks and Multimedia Communications, Springer Berlin Heidelberg, pp. 406-415, 2003.

[10] J. Ott, S. Wenger, N. Sato, C. Burmeister, and J. Rey, "Real-time transport control protocol (RTCP)-based feedback (RTP/AVPF)," IETF RFC 4585, July 2006.

[11] K. G. Murty, "Forecasting for supply chain and portfolio management," Mathematical Programming and Game Theory for Decision Making, vol. 231, 2006.

[12] The Network Simulator-ns-2. [Online]. Available: http://www.isi.edu/nsnam/ns

[13] J. Klaue, B. Rathke, and A. Wolisz, "EvalVid - A framework for video transmission and quality evaluation," in Proc. the $13^{\text {th }}$ International
Conference on Modelling Techniques and Tools for Computer Performance Evaluation, Urbana, Illinois, USA, September 2003.

[14] WiSHFUL - Wireless Software and Hardware platforms for Flexible and Unified Radio and Network Control. [Online]. Available: http://www.tkn.tu-berlin.de/research/trace/ltvt.html

[15] Z. Lifen, S. Yanlei, and L. Ju, "Performance study of transmitting MPEG4 over SCTP," in Proc. IEEE International Conference on Neural Networks \& Signal Processing, Nanjing, China, December 2003.

[16] A. Ziviani, B. E. Wolfinger, J. F. De Rezende, O. C. M. Duarte, and S. Fdida, "Joint adoption of QoS schemes for MPEG streams," Multimedia Tools and Applications, vol. 26, no. 1, pp. 59-80, May 2005.

[17] J. Niu, Y. Liu, and L. Shu, "Message dissemination in delay-tolerant networks with probabilistic encounters," Journal of Internet Technology, vol. 14, no. 3, pp. 387-400, 2013.

[18] J. L. Chen, H. C. Hsieh, and Y. T. Larosa, "Integrated LTE test-bed framework evaluating EPC compliance for IoT QoS architecture," Journal of Internet Technology, vol. 14, no. 2, 2013, pp. 171-180.

[19] J. H. Liu, Y. M. Ge, J. P. Bi, S. B. Li, and L. Guo, "Dynamic optimization model for cooperative downloading strategy of VANET," Journal of Internet Technology, vol. 14, no. 6, pp. 963-972, 2013.

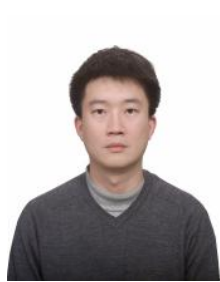

Ching-Neng Lai received his $\mathrm{PhD}$ degree in information management from National Taiwan University of Science and Technology in 2009. He joined the Faculty of the Department of Information Technology at Hsing Wu University as an assistant professor in August 2009. His research interests include performance analysis and protocol design on networks.

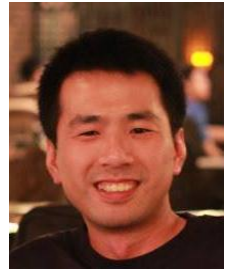

Yen-Hung Chen received his $\mathrm{PhD}$ degree at National Taiwan University of Science and Technology, in 2013. His research interests include wireless network performance analysis, protocol design, and telecommunication policy making. He currently works in the Policy Research Center, Cyber Trust Technology Institute at Institute for Information Industry. 